\title{
Influence of minor trauma to the neck on the neurological outcome in patients with ossification of the posterior longitudinal ligament (OPLL) of the cervical spine
}

\author{
S Katoh, T Ikata, N Hirai, Y Okada and K Nakauchi \\ Department of Orthopedic Surgery, School of Medicine, The University of Tokushima, 3-18-15 Kuramoto-cho, \\ Tokushima 770, Japan
}

\begin{abstract}
The influence of minor trauma to the neck on the neurological outcome in patients with ossification of the posterior longitudinal ligament (OPLL) of the cervical spine was evaluated retrospectively. Out of 118 patients treated in our clinic for cervical OPLL between 1976 and 1992, 27 had sustained minor trauma to the cervical spine. Of these 27 patients, 13 developed myelopathy, seven showed deterioration of preexisting myelopathy, and no neurological change was observed in seven patients. Regarding the relationship between the diameter of the residual spinal canal and the neurological outcome in these 27 patients, 18 out of the 19 patients with a narrow residual spinal canal $(<10 \mathrm{~mm})$ developed neurological deterioration, whereas that occurred in only two of the eight patients with a wider spinal canal $(\geqslant 10 \mathrm{~mm})$. Although the severity of myelopathy and the transverse area of the spinal cord measured from T1-weighted magnetic resonance images, in patients who had sustained minor trauma was not statistically different from patients without trauma, neurological recovery after surgical treatment was poorer in the former group than in the latter. These results indicate that even indirect minor trauma to the neck can cause irreversible changes in the spinal cord if there is marked stenosis of the cervical spinal canal; such patients who are at risk, must be educated, and should be told to avoid even minor injuries at any cost.
\end{abstract}

Keywords: ossification; posterior longitudinal ligament; trauma; neurological outcome; cervical spine injury

\section{Introduction}

The occurrence of cervical spinal cord injuries in Japan is high, and many of these patients are relatively old. ${ }^{1}$ Although epidemiological data are not yet available, we are of the opinion that the high frequency of cervical spinal stenosis either because of a developmentally stenotic spinal canal or because of ossification of the posterior longitudinal ligament (OPLL) must be important. OPLL is designated as an intractable disease by the Ministry of Public Health and Welfare of Japan, and many of these patients in Japan have a serious compression myelopathy, which is sometimes further complicated by minor trauma to the spinal cord. Although the significance of minor trauma on the neurological outcome of such patients has been widely recognised in our clinic, it has not been well reported. We have investigated the development of neurological deterioration after such minor trauma and the influence of decompression surgery regarding neurological recovery.

\section{Methods and subjects}

We reviewed the clinical records and radiographs of 118 patients who had cervical OPLL and were treated in our clinic from 1976 and 1992. There were 76 males and 42 females. The age at first presentation ranged from 35 to 78 years with a mean of 58.0 years. The mean follow-up period was 5.5 years ranging from 2 to 15 years. Forty-seven patients were treated conservatively and 71 surgically; three patients who were initially treated conservatively had surgical treatment later. Patients with major neurological deterioration immediately following major trauma were excluded from our study.

As late adverse effects have been reported in patients undergoing multisegmental anterior interbody fusion, ${ }^{2-4}$ laminoplastic posterior decompression has become the commonest surgical procedure for the treatment of patients with cervical OPLL, unless OPLL is localised to one or two segments (Figure 1). In the present series, 28 patients underwent anterior decom- 


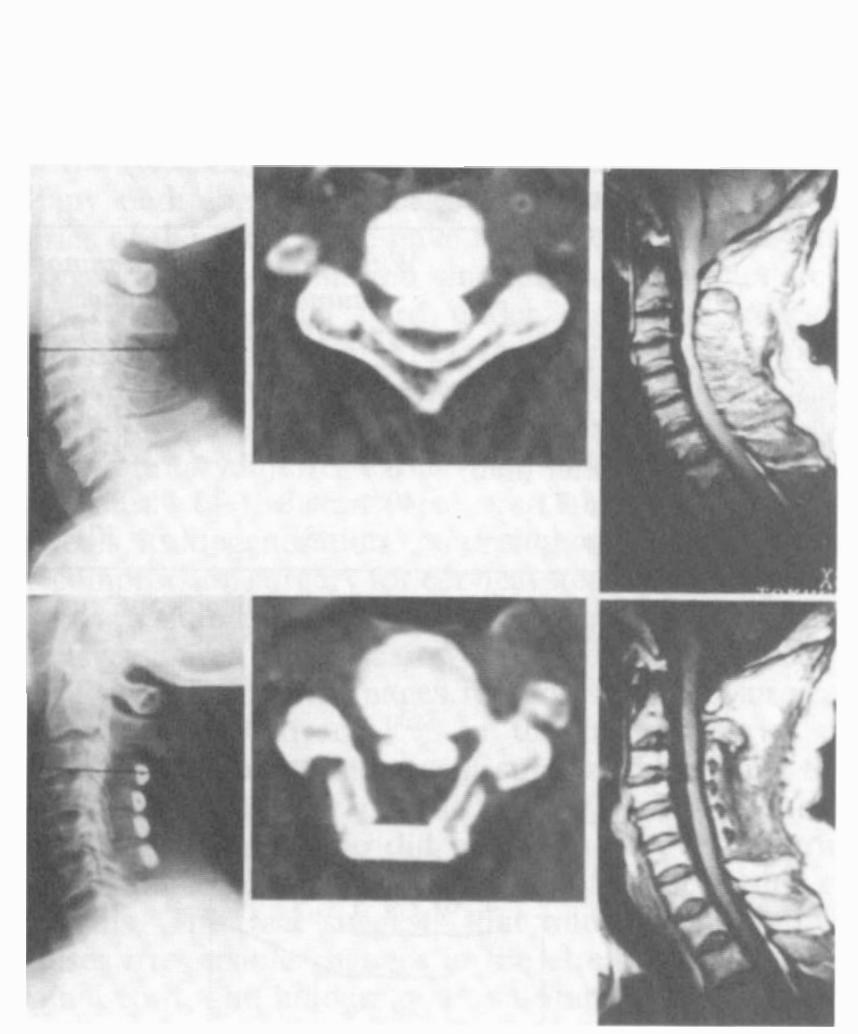

Figure 1 Illustrative case of a patient who presented with myelopathy due to OPLL and was subjected to laminoplastic posterior decompression using a hydroxyapatite interlaminar spacer $(\mathrm{a}, \mathrm{b}, \mathrm{c}$ : at presentation; d, e, f: after surgery; a, d: plain radiographs; b, e: CT; c, f: T1-weighted sagittal MR images)

pression and interbody fusion, and 43 were treated by posterior decompression procedures.

The neurological status was evaluated using the scoring system for cervical myelopathy established by the Japanese Orthopaedic Association (JOA score, Table 1). The recovery rate was calculated from the initial and the final JOA scores according to Hirabayashi's formula. ${ }^{5}$ The residual anteroposterior diameter of the spinal canal (APD) which indicates the space available for the spinal cord, was measured on plain radiographs. In 25 patients who had been investigated by magnetic resonance (MR) imaging before surgical treatment, the transverse spinal cord area on the T1-weighted axial image was also measured. ${ }^{6}$ The results obtained in patients who had sustained minor trauma and in those who had not, were compared.

\section{Results}

Twenty seven patients recalled episodes of minor neck trauma about 2 weeks previously, such as tumbling, slipping or jumping from small steps. Of the 27 patients, 13 developed myelopathy after the episode, seven experienced deterioration of pre-existing myelopathy, and one showed no change of pre-existing myelopathy. Six patients did not have, and did not develop myelopathy (Table 2). A fall on the level was the commonest cause of injury, followed by a traffic accident such as a 'whiplash'; or being struck by an object or a fall from a height of less than $2 \mathrm{~m}$ without suffering a direct injury to the neck (Table 3 ).
Table 1 Scoring system for cervical myelopathy established by the Japanese Orthopaedic Association in 1975

Motor function

Upper extremities

Unable to feed oneself with chopsticks or spoon

Can manage to feed oneself with spoon and/or fork, but not with chopsticks

Feeds oneself with chopsticks but inadequately

Feeds oneself with chopsticks but clumsily

Normal

Lower extremities

Unable to walk

Unable to walk without cane or other kind of support on a level

Walks independently on a level but needs support on stairs

Walks independently but clumsily

Normal

Sensory function

Upper extremities

Apparent sensory loss

Minimal sensory loss

Normal

Lower extremities same as upper extremities

Trunk same as upper extremities

Bladder function

Urinary retention and/or incontinence

Sense of retention and/or dribbling and/or thin stream and/or incomplete incontinence

Urinary retardation and/or pollakiuria

Normal

Table 2 Neurological changes of the patients with cervical OPLL after minor trauma

\begin{tabular}{lc}
\hline Neurological changes & $\begin{array}{c}\text { Number of } \\
\text { patients }\end{array}$ \\
\hline Developed myelopathy & 13 \\
Did not develop myelopathy & 6 \\
Deterioration of pre-existing myelopathy & 7 \\
No deterioration of pre-existing myelopathy & 1 \\
Total & 27 \\
\hline
\end{tabular}

Table 3 Causes of minor trauma

\begin{tabular}{lc}
\hline Causes of minor trauma & $\begin{array}{c}\text { Number of } \\
\text { patients }\end{array}$ \\
\hline Fall on level & 12 \\
Traffic accident & 6 \\
Being struck by an object & 5 \\
Fall from a height (less than 2 m) & 4 \\
Total & 27 \\
\hline
\end{tabular}

Regarding the relationship between the residual AP diameter (APD) and neurological changes, deterioration of myelopathy after minor trauma was observed in 18 of 19 patients whose APD was less than $10 \mathrm{~mm}$. On 
the other hand, only two out of eight patients whose APD was $10 \mathrm{~mm}$ or more had neurological deterioration after minor trauma (Table 4). There was a statistically significant difference between these two groups (Fisher's exact probability test, $P<0.01$ ).

Spontaneous neurological deterioration was also found in patients who had not suffered any kind of trauma, although such an occurrence was rare. Among those without trauma, 16 patients whose APD was less than $10 \mathrm{~mm}$ and who had been treated conservatively, five showed spontaneous neurological deterioration mainly due to progression of OPLL itself, whereas only two out of 34 patients whose APD was $10 \mathrm{~mm}$ or more, had neurological deterioration, (Fisher's exact probability test, $P<0.05)$.

Regarding patients who were treated surgically, there were no statistically significant differences in mean age, mean duration of illness, mean spinal cord area measured from T1-weighted axial images or mean JOA scores before surgery between patients $(n=19)$ who had suffered minor neck trauma and those $(n=52)$ who had not (Table 5). Neurological recovery after surgical treatment was poorer in the former group than in the latter (Figure 2). The JOA score of those two groups at follow-up more than 1 year after surgery was $11.7 \pm 3.2$ in patients who had had trauma, and $13.7 \pm 2.7$ in those without a history of trauma. The recovery rate was $42.0 \%$ in patients with trauma, and $61.7 \%$ in those without trauma. These differences in JOA scores and recovery rates were statistically significant $(P<0.05$, unpaired $t$ test $)$.

\section{Discussion}

Ossification of the posterior longitudinal ligament (OPLL) in the thoracic and lumbar spine was first reported by Key in $1838 .^{7}$ Cervical myelopathy as a consequence of OPLL was reported by Tsukimoto in $1960{ }^{8}$ and there has been an increasing number of reports on this condition. According to the authoritative paper by Tsuyama, the incidence of OPLL in eastern Asiatic subjects is about $2 \%$, while that in white subjects is $0.16 \% .^{9}$

OPLL is categorised as an intractable disease by the Japanese government. The pathogenesis has not yet been elucidated, although it is regarded as a different pathological process from that of calcification of the ligamentum flavum; and genetic, metabolic and dietary

Table 4 Relationship between residual spinal canal diameter and neurological changes in patients who suffered minor trauma

\begin{tabular}{lccc}
\hline $\begin{array}{l}\text { Residual } \\
\text { antiposterior } \\
\text { diameter of the } \\
\text { spinal cord }\end{array}$ & $\begin{array}{c}\text { Development } \\
\text { or deterioration } \\
\text { of myelopathy }\end{array}$ & Unchanged & Total \\
\hline$<10 \mathrm{~mm}$ & 18 & 1 & \\
$\geqslant 10 \mathrm{~mm}$ & 2 & 6 & 19 \\
Total & 20 & 7 & 27 \\
\hline
\end{tabular}

Table 5 Background of the patients with myelopathy due to OPLL who were treated surgically

\begin{tabular}{lcc}
\hline & $\begin{array}{c}\text { With minor } \\
\text { trauma } \\
(n=19)\end{array}$ & $\begin{array}{c}\text { Without minor } \\
\text { trauma } \\
(n=52)\end{array}$ \\
\hline Age (years) & $57.9 \pm 7.0$ & $55.5 \pm 10.0^{\mathrm{NS}}$ \\
Duration of illness (months) & $49.3 \pm 42.5$ & $44.6 \pm 45.5^{\mathrm{NS}}$ \\
Residual AP diameter (mm) & $6.7 \pm 1.7$ & $7.2 \pm 1.9^{\mathrm{NS}}$ \\
Spinal cord area on T1- & $40.4 \pm 6.8$ & $42.4 \pm 8.3^{\mathrm{NS}}$ \\
weighted MR image $\left(\mathrm{mm}^{2}\right)$ & $(n=8)$ & $(n=17)$ \\
\hline
\end{tabular}

Mean \pm standard deviation; NS: not significant using unpaired $t$ test

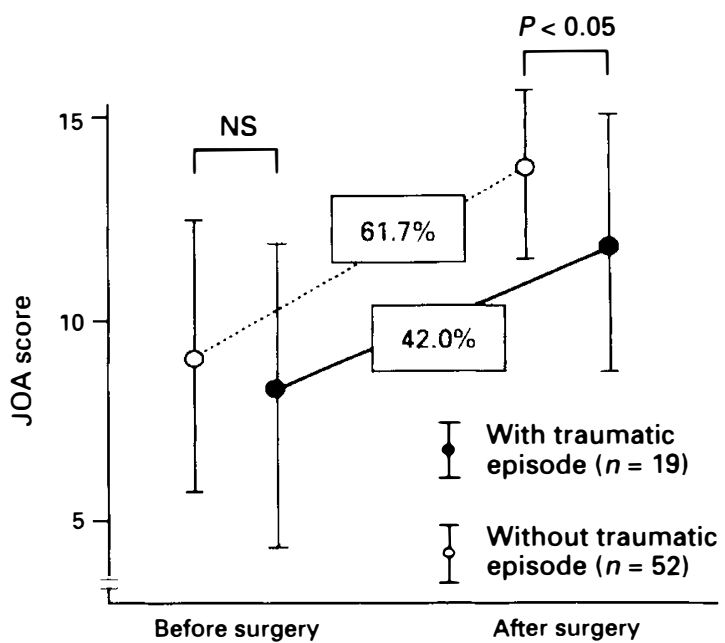

Figure 2 Linegraphs demonstrating the surgical outcome in patients who had suffered a minor trauma and those who had not. Recovery rates of both groups, which are shown in the rectangles, were significantly different (unpaired $t$ test)

factors of the patients as well as the local condition of the ligament have been investigated..$^{10-13}$ It is widely recognised that OPLL is a common cause of spinal canal stenosis, sometimes with myelopathy and/or radiculopathy. Considerable knowledge on the development of neurological deficits in relation to the configuration or shape of the ossified segments has been accumulated as a result of detailed autopsy studies and progressive radiological evaluations including MRI. ${ }^{6,14}$ However, arterial occlusion or other causes of myelopathy have not yet been clearly appreciated.

The present study has demonstrated that cervical myelopathy developed or, if already present, deteriorated after minor neck trauma such as a fall on level ground in $16.9 \%$ of patients with OPLL, which is comparable to the incidence of $20.9 \%$ reported by Tsuyama. ${ }^{9}$ It is not difficult to postulate that the narrowness of the space available for the spinal cord is a critical factor for neurological deterioration immediately after a neck injury since a reduction of sagittal spinal canal size contributes significantly to the severity of the neurological deficit after a spinal injury. ${ }^{15}$ Neurological deterioration was delayed for several days after minor neck trauma in half of the patients in the present series. This delay may be similar to the delay of 
neurological deterioration after a spinal cord injury. ${ }^{16,17}$ Many pathological processes secondary to direct injuries of the spinal cord have been postulated, such as oxygen radical formation and eicosanoids metabolism. ${ }^{18-21}$ The spinal cord under markedly stenotic conditions $(<10 \mathrm{~mm}$ of APD) must be vulnerable even to indirect injuries to the neck, and secondary pathological processes may occur after clinically insignificant trivial primary injuries to the vulnerable spinal cord.

A study of the transverse area of the spinal cord reveals its degeneration. Neurological recovery after decompression surgery for cervical stenotic myelopathy shows a statistically significant correlation with the transverse area of the narrowest part of the spinal cord on T1-weighted MR images regardless of the surgical procedures employed. ${ }^{6,14,22}$ In the present series in those patients who underwent surgical treatment, the area was not different in those who suffered minor neck trauma and those who did not; however, the neurological outcome was poorer in the former group of patients. This fact suggests that minor neck trauma causes irreversible changes in the already compressed spinal cord, and hinders potential improvement of the neurological symptoms after surgical decompression.

There appears to be general agreement in Japan that surgical treatment should not be employed for patients with a stenotic spinal canal if there are no neurological symptoms. Once symptoms of long-tract involvement develop, our practice is to operate, usually employing a laminoplastic procedure. For patients who develop myelopathy after minor neck trauma, we also undertake a surgical decompression not only to treat preexisting myelopathy, but also to prevent neurological deterioration by further trauma. Patients who have OPLL are strongly advised to avoid all such injuries.

\section{Acknowledgement}

This work was supported in part by a grant from the investigation committee on the ossification of the spinal ligaments of the Japanese Ministry of Public Health and Welfare.

\section{References}

1 Shingu H, Ikata T, Katoh S, Akatsu T. Spinal cord injuries in Japan: A nationwide epidemiological survey in 1990. Paraplegia 1994; 32: 3-8.
2 Goto S, Mochizuki M, Watanabe T et al. Long-term follow-up study of anterior surgery for cervical spondylotic myelopathy with special reference to the magnetic resonance imaging findings in 52 cases. Clin Orthop 1993; 291: 142-153.

3 Gruss P, Tannenbaum H. Stress exertion on adjacent segments after ventral cervical fusion. Arch Orthop Trauma Surg 1983; 101: 283-286.

4 Yonenobu $\mathrm{K}$ et al. Causes of neurologic deterioration following surgical treatment of cervical myelopathy. Spine 1986; 11: $818-823$.

5 Satomi K, Nishu Y, Kohno T, Hirabayashi K. Long-term follow-up studies of open-door expansive laminoplasty for cervical stenotic myelopathy. Spine 1994; 19: 507-510.

6 Okada Y et al. Magnetic resonance imaging study on the results of surgery for cervical compression myelopathy. Spine 1993; 18: 2024-2029.

7 Key CA. On paraplegia depending on disease of the ligaments of the spine. Guy's Hosp Rep 1838; 3: 17-34.

8 Tsukimoto $\mathrm{H}$. On an autopsied case of compression myelopathy with a callus formation in the cervical spinal canal (in Japanese). Nihon-geka-hokan 1960; 29: 1003-1007.

9 Tsuyama N. Ossification of the posterior longitudinal ligament of the spine. Clin Orthop 1984; 184: 71-84.

10 Sakamoto $\mathrm{R}$ et al. Comparative study between magnetic resonance imaging and histopathologic findings in ossification or calcification of ligaments. Spine 1991; 16: 1253-1261.

11 Sakou $\mathrm{T}$ et al. Genetic study of ossification of the posterior longitudinal ligament in the cervical spine with human leukocyte antigen haplotype. Spine 1991; 16: 1249-1252.

12 Seichi A, Hoshino Y, Ohnishi I, Kurokawa T. The role of calcium metabolism abnormalities in the development of ossification of the posterior longitudinal ligament of the cervical spine. Spine 1992; 17: S30-32.

13 Terayama K. Genetic studies on ossification of the posterior longitudinal ligament of the spine. Spine 1989; 14: 1184-1191.

14 Fujiwara $\mathrm{K}$ et al. Morphometry of the cervical spinal cord and its relation to pathology in cases with compression myelopathy. Spine 1988; 13: 1212-1216.

15 Eismont FJ, Clifford S, Goldberg M, Green B. Cervical sagittal spinal canal size in spine injury. Spine 1984; 9: 663-666.

16 Frankel HL. Ascending cord lesion in the early stages following spinal injury. Paraplegia 1969; 7: 111-118.

17 Katoh S, El Masry WS. Neurological recovery after conservative treatment of cervical cord injuries. J Bone Joint Surg $\mathrm{Br}$ 1994; 76: 225-228.

18 Janssen L, Hansebout RR. Pathogenesis of spinal cord injury and newer treatments. A review. Spine 1989; 14: 23-32.

19 Iwasa K, Ikata T, Fukuzawa K. Protective effect of vitamin E on spinal cord injury by compression and concurrent lipid peroxidation. Free Radical Biol Med 1989; 6: 599-606.

20 Mitsuhashi $\mathrm{T}$ et al. Increased production of eicosanoids, $\mathrm{TXA}_{2}$, $\mathrm{PGI}_{2}$ and $\mathrm{LTC}_{4}$ in experimental spinal cord injuries. Paraplegia 1994; 32: 524-530

21 Taoka Y, Ikata T, Fukuzawa K. Influence of dietary vitamin E deficiency on compression injury of rat spinal cord. J Nutr Sci Vitamin 1990; 36: 217-226.

22 Okada Y, Ikata T, Yamada H, Katoh S. Morphological analysis of the cervical spinal cord, dural tube, and spinal canal by magnetic resonance imaging in normal adults and patients with cervical spondylotic myelopathy. Spine 1994; 19: 2331-2335. 